\title{
Habitação saudável no Programa Saúde da Família (PSF): uma estratégia para as políticas públicas de saúde e ambiente
}

\author{
Healthy house in the Family Health Program (PSF): \\ a strategy for the health and environment \\ public politics
}

Simone Cynamon Cohen 1

Szachna Eliasz Cynamon ${ }^{1}$

Débora Cynamon Kligerman 1

Rafaela Facchetti Assumpção 1

\footnotetext{
1 Departamento

de Saneamento e Saúde

Ambiental, Escola Nacional

Abstract Scientific evidences show that the health is linked with the way of people life and environment interactions. It is not only a genetic and biology determinism. In that way, housing is the basic locus of Family Health Promotion. Healthy house's conception has focused in improvement of quality of life. The challenge is around the strengthen of health specific understanding in built space as human biology, environment and life styles. Those factors are the main cause of illness and death inside house. To face that challenge it's necessary to articulate public policy in housing, health, environment and urban infrastructure and in the way of intercessory alliances in holistic, integrated and multidisciplinary vision. Therefore, the Family Health Program as Health Promotion strategy, at local level is the beginning point of intervention and articulation between two methodology: Family Health Program and Healthy Housing.

Key words Healthy Housing, Family Health Program, Health promotion, Public policy in health and environment
Resumo Evidências científicas apontam que a saúde está relacionada ao modo de viver das pessoas e suas interações com o meio ambiente e não somente com a idéia hegemônica do determinismo biológico e genético. Nesse sentido, a habitação aparece como lócus base de Promoção da Saúde Familiar. O conceito de habitação saudável possui como ponto central a preocupação com o processo gradual de melhoria da qualidade de vida. O desafio está na consolidação da intervenção sobre os fatores determinantes da saúde no espaço construído, entendendo-os como biologia humana, meio ambiente e estilos de vida. Sendo estes fatores, na habitação, as principais causas de enfermidade e morte. Para enfrentar esse desafio é necessário articular as políticas públicas de habitação, de saúde, de ambiente e de infra-estrutura urbana e formar alianças intersetoriais, em uma visão holística, integradora e multidisciplinar. Portanto, o Programa Saúde da Família como estratégia de Promoção da Saúde em nível local passa a ser o ponto de intervenção e início de articulação entre as duas metodologias: Programa Saúde da Família e Habitação Saudável.

Palavras-chave Habitação Saudável, Programa Saúde da Família, Promoção da Saúde, Políticas públicas em saúde e ambiente 


\section{Introdução}

No Brasil a situação ambiental está em contínuo processo de degradação, ocorrendo: a transição epidemiológica marcada pela prevalência das condições de morbi-mortalidade, verificadas em alguns setores; a permanência de certas doenças infecto-parasitárias devido à falta de infra-estrutura urbana básica; o crescimento de doenças crônicas não infecciosas; o aparecimento de novas doenças sexualmente transmissíveis; e o crescimento da violência urbana devido ao uso de drogas e a outros fatores comportamentais, como o stress da vida cotidiana.

O Estado brasileiro ainda se apresenta muito dividido por setores e fragmentado em suas ações, desvinculando o social do econômico. Porém, através de esforços da sociedade em redemocratizar o País, foram alcançados muitos avanços em 1988, como a elaboração da atual Constituição e a criação do Sistema Único de Saúde.

A política de saúde construída no Brasil, a partir desse ano, vem contribuindo para a melhoria da qualificação do Sistema Único de Saúde, tendo em seu arcabouço princípios de Promoção da Saúde como universalidade, integralidade e eqüidade das diretrizes de descentralização e organização hierarquizada, que constitui uma visão ampliada no nível nacional e tem como ponto central sua preocupação com o processo gradual de melhoria da qualidade de vida.

Tal melhoria só é efetiva através da utilização de estratégias e iniciativas capazes de operacionalizar sua interação por intermédio da elaboração de políticas públicas saudáveis, que exigem a ação intersetorial e de uma nova institucionalidade social. Esta se materializa através de propostas como a Estratégia da Saúde da Família, que tem em sua lógica central a operacionalização de conceitos como a territorialização, vinculação, responsabilização e resolutividade com um olhar integral sobre o ambiente em suas dimensões físicas, socioculturais, biopsicossociais, nas quais estão inseridos os indivíduos e suas famílias. Necessita incorporar às suas práticas conceitos como a integralidade da atenção à saúde e a perspectiva da troca entre saberes técnico-científicos e o saber popular, com o qual a comunidade se identifica e está dele imbuída.

Outras iniciativas que operacionalizam os princípios da Promoção da Saúde são: municí- pios saudáveis, vigilância ambiental em saúde, melhoria sanitária domiciliar, Agenda 21, desenvolvimento local integrado/sustentável e rede brasileira de habitação saudável.

Responsável por este projeto, a Rede Brasileira de Habitação Saudável (RBHS) é uma ferramenta para operacionalização da política de promoção de saúde no âmbito da habitação. Sua estratégia se baseia no enfoque intersetorial, multidisciplinar, na participação comunitária e na aliança em rede. Seu propósito é identificar, avaliar e manejar a questão da habitação saudável na esfera local. Para tanto, desenvolve um plano de atividades baseado na construção de capacidades, projetos e desenvolvimento de pesquisa, serviços técnico-científicos e intervenção comunitária, bem como no desenvolvimento de metodologias e procedimentos, na implementação da vigilância epidemiológica e ambiental em saúde.

A precariedade habitacional, deterioração da qualidade de vida, impacto na saúde de ambientes insalubres e o distanciamento da comunidade científica da realidade comprovam a necessidade de aumentar a eficácia e eficiência das políticas públicas de saúde. Para isso, foram feitos acordos, estabelecidas alianças e propostas estratégicas para concentrar esforços e recursos a partir das potencialidades das instituições acadêmicas e públicas envolvidas com as questões de saúde e habitação.

Neste contexto se inclui a proposta da incorporação do conceito de habitação saudável da RBHS com a política pública de saúde, a partir de seu grande número de pontos afins.

\section{Justificativa da proposta}

Hoje, a população urbana no Brasil chega a $80 \%$ da população total, sendo que $50 \%$ vivem em áreas de favela. Os 10\% mais ricos no Brasil têm renda 22 vezes maior que $40 \%$ dos mais pobres. Entretanto, se compararmos o Brasil com a América Latina, esta cifra é 19 vezes maior, o que comprova que em relação à América Latina, o Brasil apresenta maiores contrastes (Cepal, 2000) onde $80 \%$ dos mais ricos possuem saneamento básico, enquanto $32 \%$ dos $40 \%$ mais pobres não têm esse benefício (MS, 2002).

A partir do III Fórum Social Mundial foram identificados problemas que só poderiam ser solucionados com a cooperação entre diversos atores sociais como as instituições acadêmicas, ONGs, movimentos sociais e órgãos 
governamentais que interagiram na intenção de implementar um conceito abrangente de política pública de habitação saudável.

A precariedade habitacional, a deterioração da qualidade de vida, o impacto na saúde de ambientes insalubres e o distanciamento da comunidade científica da realidade comprovaram a necessidade de aumentar a eficácia e eficiência das políticas públicas de saúde.

Do ponto de vista do paradigma do ambiente como determinante da saúde, a habitação se constitui em um espaço de construção da saúde e consolidação do seu desenvolvimento. A família tem seu assento na habitação e, com isto, a habitação é o espaço essencial, o veículo da construção e desenvolvimento da Saúde da Família.

Para o enfrentamento do desafio da consolidação da intervenção sobre os fatores determinantes da saúde no espaço construído, fazse necessária a articulação das políticas públicas, de habitação, saúde, meio ambiente e infra-estrutura urbana. O que convoca a formação de alianças intersetoriais em uma visão holística integradora e multidisciplinar, em que a participação comunitária tem um papel essencial para o enfrentamento das questões locais da precariedade das relações do meio ambiente e o homem em seu habitar.

Ao identificar que o Programa Saúde da Família responde ao problema discutido no III Fórum Social Mundial e constitui uma prioridade da política de saúde pública do Brasil e da Organização Pan-Americana de Saúde - OPAS, valoriza-se a iniciativa da Habitação Saudável, como ferramenta para otimização dos resultados do Programa Saúde da Família.

Propõe-se, então, uma aliança estratégica para contribuir e alcançar os objetivos da saúde familiar, através do envolvimento da iniciativa de habitação saudável por intermédio da rede brasileira de habitação saudável com o apoio da rede interamericana.

\section{Marcos conceituais de saúde, ambiente e habitação}

O entendimento da habitação como um espaço onde a função principal é ter a qualidade de ser habitável faz com que uma análise incorpore a visão das múltiplas dimensões que compõem a habitação: cultural, econômica, ecológica e de saúde humana. A concepção integradora da habitação considera os usos que fazem da mes- ma os habitantes, incluindo os estilos de vida e condutas de risco; é, portanto, uma concepção sociológica, devendo o conceito habitação saudável incluir o seu entorno, como ambiente, e agenda da saúde de seus moradores (OPAS, 2000).

Como metodologia, o conceito de habitação saudável se aplica desde o ato da elaboração do seu desenho, microlocalização e construção, estendendo-se até seu uso e manutenção. Está relacionado com o território geográfico e social onde a habitação se assenta, os materiais usados para sua construção, a segurança e qualidade dos elementos combinados, o processo construtivo, a composição espacial, a qualidade dos acabamentos, o contexto global do entorno (comunicações, energia, vizinhança) e a educação em saúde ambiental de seus moradores sobre estilos e condições de vida saudável (Cohen, 2003).

O conceito de ambiente e entorno saudável incorpora a necessidade de ter equipamentos urbanos básicos com saneamento, espaços físicos limpos e estruturalmente adequados e redes de apoio para se conseguir hábitos psicossociais sãos e seguros, isentos de violência (abuso físico, verbal e emocional) (Cohen, 2003).

A idéia de municípios/cidades saudáveis só é possível se houver uma política de habitação saudável. Portanto, a estratégia de habitação saudável se insere através da colaboração interprogramática em municípios/cidades saudáveis.

Entende-se por padrão de habitabilidade a adoção de tipologias em correspondência aos requisitos mínimos que garantam o morar com desfrute de saúde e bem-estar e propiciem a dignidade humana. Nesse sentido, promove o pleno exercício do ato de morar, ampliando e melhorando, respectivamente, a qualidade do espaço e da vida. Padrões que propiciem o convívio harmônico através da reflexão e do aprimoramento do lugar/objeto/habitação.

Segundo a OPAS (2000), entende-se por fatores de risco o espectro de causalidades que têm a possibilidade de interferir nos sistemas vivos, psicossociais e do ser humano em seu funcionamento, com prejuízo às condições individuais ou coletivas de saúde. $\mathrm{Na}$ análise de fatores de risco são considerados os aspectos: físicos, químicos, psicossociais, biológicos, socioeconômicos e sindrômicos.

Outro conceito importante para ser desenvolvido é o da atenção primária ambiental, con- 
siderada uma estratégia de ação ambiental preventiva e participativa, que reconhece o direito das pessoas a viverem em um meio ambiente saudável e de serem informadas sobre os riscos ambientais em relação a sua saúde e bem-estar (Teixeira, 1997).

Encontra-se no âmbito da atenção primária aquelas medidas de proteção ou recuperação de baixa complexidade, denominadas ações ambientais primárias, tais como: educação ambiental; contaminação simples dos ambientes de trabalho e domicílios; poluição sonora; qualidade e disponibilidade de água potável; controle de vetores transmissores de doenças, em combinação com a vigilância epidemiológica; manejo e reciclagem de resíduos sólidos; controle de qualidade de alimentos, em combinação com a vigilância sanitária; erosão de solos, queimadas florestais, pragas, urbanização de ruas, áreas verdes e outras (Buss et al., 1998). Através deste enfoque, a atenção primária ambiental aplica sua estratégia na área de habitação saudável.

Outro conceito importante para ser desenvolvido no campo da saúde e da habitação é o de vigilância ambiental em saúde. Por definição, a vigilância ambiental em saúde se configura como um conjunto de ações que proporcionem o conhecimento e a detecção de qualquer mudança nos fatores determinantes e condicionantes do meio ambiente que interferem na saúde humana, com a finalidade de recomendar e adotar as medidas de prevenção e controle dos fatores de riscos e das doenças ou agravos relacionados à variável ambiental (www.funasa.gov.br). A vigilância ambiental em saúde se aplica no âmbito da habitação saudável, a partir do monitoramento ambiental e estabelecimento de valores-limite de exposição para estressores ambientais e conduzem a uma proposta de medidas de intervenção e controle para otimização sanitária do ambiente.

A Carta de Ottawa define a promoção da saúde como o processo de capacitação da comunidade para atuar na melhoria da sua qualidade de vida e saúde, incluindo uma maior participação no controle deste processo (Buss, 2000 apud WHO, 1986). A habitação é o espaço principal da promoção da saúde na comunidade.

No processo de incremento da qualidade de vida, sobretudo em formações sociais com alta desigualdade sociossanitária é importante que sejam mostrados movimentos que concretizem, através de seus próprios fundamentos e práticas, a idéia da promoção da saúde. Um deles é o das cidades saudáveis, surgido na Euro- pa, em 1986, no mesmo ano da Conferência Internacional sobre Promoção da Saúde, que ocorreu em Ottawa, Canadá. O conceito de município saudável é uma proposta de promoção da saúde, cujos moldes foram delineados na Carta de Ottawa (1986). A idéia de municípios/cidades saudáveis só é viável se houver uma política de habitação saudável, pois não é possível encontrar um município/cidade sem a existência de habitações saudáveis. Portanto, a estratégia de habitação saudável se insere através da colaboração interprogramática em municípios/cidades saudáveis. Segundo a Organização Pan-Americana de Saúde - OPAS, o município/cidade saudável é uma estratégia que permite fortalecer a execução das atividades de Promoção da Saúde como a mais alta prioridade em uma agenda política local, desenvolvendo planos de ação baseados nos princípios de saúde para todos da OMS.

\section{Metodologia da proposta de articulação RBHS-PSF (lócus operacional) e possíveis produtos}

A proposta RBHS é de articular o Ministério da Saúde e Secretarias de Estado que desenvolvem o Programa Saúde da Família, das Escolas Técnicas do SUS, equipes de trabalho do PROFAE/MS envolvidas na Formulação dos Currículos por Competência para Auxiliares e Técnicos de Enfermagem e na Modernização da Rede de Escolas Técnicas do SUS, com a Rede Brasileira de Habitação Saudável - Fiocruz/Rede Amazônica de Habitação Saudável - Unama/ Rede Sudeste de Habitação Saudável - Fiocruz/Red Interamericana de Vivienda Saludable - OPAS, onde todos trabalham na lógica da Promoção da Saúde e podem cooperar para que o PSF se transforme em lócus operacional da RBHS / RAHS / RSHS realizando as etapas a seguir enumeradas.

\section{Primeira etapa: Identificação de áreas-piloto}

- Encontros do grupo técnico da RBHS com os técnicos do Programa da Saúde da Família da Secretaria Municipal de Saúde da Cidade do Rio de Janeiro para escolha de três áreas-piloto. - Divulgação da proposta do projeto e identificação de três áreas para experimento piloto em três comunidades, sendo duas em favelas (espaço urbano/periurbano) e a terceira em 
área de loteamentos irregulares (ocupações rurais precários próxima ao espaço periurbano). As duas primeiras (favelas), com características distintas, uma em ocupação em encosta e outra em ocupação em área de mangue e em planície. Dessa forma, teríamos a evidência científica que a proposta poderia ser lançada, depois de obter os resultados em outros lugares do território nacional. Inicialmente pensou-se na área do Complexo de Manguinhos (planície); Complexo do Caricó ou Complexo do Alemão (aclive) e Pinheiral.

\section{Segunda etapa: \\ Elaboração de oficinas}

- Elaboração de duas oficinas, uma em nível nacional, em agosto de 2003, e outra em nível internacional em setembro de 2003. A oficina de agosto "Habitação saudável \& família saudável” teve como objetivo geral desenvolver o projeto "Habitações Saudáveis no SUS, uma Estratégia de Ação para o PSF: uma Incorporação do Conceito de Habitação Saudável na Política Pública de Saúde", através da interação das instituições afiliadas à Rede Brasileira de Habitação Saudável nos campos da Habitação Saudável \& Família Saudável e objetivos específicos: 1) desenvolver e debater conteúdo programático para cartilhas Habitação Saudável \& Família Saudável destinadas aos agentes comunitários em saúde, técnicos em saúde e técnicos na área de melhoria sanitária domiciliar; 2) desenvolver e debater conteúdo mínimo para programa de centro de atenção em saúde, ambiente e habitação (módulo do PSF) “educativamente saudável”, que incorpore conceito de habitação saudável; 3 ) desenvolver e debater conteúdo mínimo para curso de capacitação e educação continuada para agentes e técnicos em saúde que incorpore o conceito de habitação saudável.

A oficina de setembro "Vivienda saludable \& família saludable" teve como objetivo geral divulgar a proposta de Vivienda Saludable e Saúde da Família como políticas de saúde no Brasil, e objetivos específicos: 1) refletir sobre o desenho do projeto Vivienda Saludable e Saúde da Família sob os enfoques: teórico, funcional, das minorias e da política nacional de saúde; 2) refletir sobre a cooperação técnica Inter-Regional na Vivienda Saludable na visão da OPS, na visão da Red Interamericana e oportunidades de cooperação entre América e Europa na vivienda saludable e atividades do Centro Europeo de Saneamento Ambiental de EURO/OMS.

\section{Terceira etapa: \\ Elaboração de projeto}

- Realização de Encontros para a integração interna da proposta na Rede Brasileira de Habitação Saudável com os núcleos regionais Rede Amazônica de Habitação Saudável e Rede Sudeste de Habitação Saudável.

- Integração externa da proposta da RBHS, acompanhada pela Rede Interamericana/OPAS com o Sistema Único de Saúde/Programa da Saúde da Família - Ministério da Saúde/Fiocruz, Funasa, Unama, SMH-PCRJ, entre outros.

- Experiências piloto utilizadas para estruturar o modelo de capacitação/educação continuada, testar e avaliar o modelo, em um processo de construção de indicadores.

\section{Quarta etapa: \\ Execução do projeto}

- Desenvolvimento de material didático e educativo para dar suporte ao início dos trabalhos - guia metodológico de repasse de conhecimento/informação para agentes comunitários em saúde que incorporem o conceito e metodologia da habitação saudável.

- Capacitação e educação continuada dos agentes de saúde ligados ao PSF (Programa Saúde da Família) em saúde, habitação e ambiente.

- Elaboração de proposta e edificação de Posto de Atenção Básica e Primária Ambiental nas referidas comunidades.

- Pesquisa Investigativa

Linha 1: investigar qual a influência do ambiente da habitação no quadro de morbi-mortalidade no Brasil;

objetivo: apontar alternativas de melhorias que possam ser executadas para interromper o processo de transmissão das doenças.

Linha 2: estudo dirigido ao diagnóstico local de habitabilidade e verificação (classificação) dos padrões existentes;

objetivo: estabelecer as bases de um sistema de vigilância epidemiológica ambiental para a habitação a partir da família;

Linha 3: "cruzamento de variáveis" (Fatores de Risco à Saúde Presentes na Habitação X Padrão de habitabilidade);

objetivo: realização de um plano piloto de intervenção, incluindo propostas de melhorias dirigidas à promoção da saúde e incorporação de padrões e modelos adequados de habitabilidade no local. 


\section{Quinta etapa: Produtos esperados}

- Elaboração de um banco de dados sobre doenças e fatores de risco relacionado ao ambiente habitacional;

- Cartilha Habitação Saudável \& Família Saudável;

- Programa mínimo do Centro de Atenção em Saúde e Ambiente;

- Programa mínimo de capacitação e educação continuada para agentes de saúde e técnicos de saúde que incorpore o conceito de habitação saudável;

- Oficinas temáticas desenvolvidas para fortalecimento do produto;

- Guia para análise de projetos habitacionais do ponto de vista de habitação saudável;

- Guia para análises dos fatores de risco à saúde da família, evitando doenças relacionadas a ambientes habitacionais precários;

- Elaboração de normas construtivas para criação de ambientes saudáveis para essas áreas.

\section{Sexta etapa:}

Resultados esperados

- Esclarecimento dos fatores etiológicos de risco das enfermidades relacionadas à habitação nestas áreas escolhidas;

- Proposta de um Sistema de Vigilância Epidemiológica e Ambiental para habitação nas áreas escolhidas;

- Elaboração de um banco de dados sobre doenças e fatores de risco relacionados ao ambiente habitacional nestas áreas;

- Capacitação de profissionais (agentes comunitários e técnicos) na área de habitação e saúde da família;

- Avaliação e Propostas de Políticas Públicas em correspondências com os resultados;

- Identificação dos Padrões Regionais de Habitabilidade existente nestas áreas e proposição de critérios preventivos e corretivos para a saúde;

- Elaboração de perfil habitacional regional para essas áreas.

\section{Sétima etapa:}

Elaboração de workshop

- Elaboração do relatório final;

- Identificação de novas localidades e multiplicação da experiência obtida;

- Desenvolvimento de critérios de sustentabilidade a partir de debate em workshop com os políticos, técnicos e comunidades envolvidas no projeto.

\section{Considerações finais}

Pensa-se ser esta proposta um grande passo para a inclusão do conceito e da metodologia de habitação saudável nas políticas públicas de saúde, através do Programa da Saúde da Família.

O fortalecimento do produto esperado (modelo de capacitação/educação continuada de políticas públicas de saúde/conceito e metodologia da habitação saudável) dependerá do Ministério da Saúde, através da definição de habilidades e competências específicas aos profissionais de saúde.

O desenvolvimento de guia metodológico de repasse de conhecimento/informação como material didático e educativo, elaborado em escala, servirá para dar suporte ao processo de capacitação e educação continuada para agentes comunitários e técnicos em saúde do Programa da Saúde da Família nas áreas de saúde, ambiente e habitação.

Os Postos de Atenção Básica e Primária Ambiental nas referidas comunidades serão o lócus operacional de integração do PSF com a RBHS.

Ao longo do desenvolvimento do projeto serão realizadas oficinas temáticas para o fortalecimento dos produtos.

Pretende-se com o desenrolar do projeto o aprofundamento de um estudo sobre habitabilidade nestas áreas e o desenvolvimento de indicadores na área de saúde, ambiente e habitação que sejam interligados.

Em próxima etapa, dando continuidade ao projeto nestas áreas, também se pretende coletar dados, identificar e mapear áreas de risco habitacional, sanitário e ambiental, através do projeto Sistema Integrado de Monitoramento Ambiental (SIMA).

Com o processo de avaliação desta proposta, pretende-se criar um escritório-modelo da RBHS, que deverá trabalhar com ações corretivas (como melhorias domiciliares e regularização fundiária e urbanística) e preventivas (propostas urbanísticas, projetos em habitação saudável e de desenvolvimento comunitário).

Pretende-se também o estímulo à produção intelectual através de incentivo à pesquisa (coleta e canalização de recursos).

Com o aprendizado desta proposta pretende-se também o desenvolvimento de curso de 
especialização em habitação, saúde e ambiente em universidades e instituições de pesquisa, através de convênio firmado da RBHS/ESCGOV/ENSP/Fiocruz com as referidas instituições.

A sustentabilidade deste processo perpassa pelo ensino, como uma contribuição do sistema de saúde, que deve preparar seus técnicos para gerência de riscos ambientais e o trabalho social com a família, incorporando no currículo dos técnicos de saúde o conceito, a metodologia e procedimento da Habitação Saudável, assim como sua aplicação na Estratégia da Saúde da Família.

\section{Colaboradores}

SC Cohen trabalhou nos marcos conceituais, embasamento da proposta, parte metodológica, referências bibliográficas e articulação no nível político-institucional.

SE Cynamon, DC Kligerman e RF Assumpção trabalharam na metodologia da proposta de articulação da Rede Brasileira de Habitação Saudável e o Programa da Saúde da Família e nas referências bibliográficas.

\section{Referências bibliográficas}

Buss PM et al. 1998. Promoção da Saúde e Saúde Pública. ENSP, Rio de Janeiro.

Buss PM 2000. Qualidade de vida e saúde. Abrasco, RecifeSão Paulo.volume 5 número 1.

Cepal 2000. Panorama Social da América Latina 1999: Agenda Social. Cepal, Santiago do Chile.

Cohen SC 2000. Habitação e saúde como instrumentos de políticas públicas saudáveis e de concretização de uma estratégia de promoção de saúde.Trabalho final da disciplina Introdução à Promoção de Saúde, de doutorado em Saúde Pública. Escola Nacional de Saúde Pública, Rio de Janeiro.

Cohen SC et al. 2003. Habitação saudável no SUS. Uma estratégia de ação para o PSF: uma incorporação do conceito de habitação saudável na política pública de saúde. Documento produzido pela Rede Brasileira de Habitação Saudável. ENSP, Rio de Janeiro.

Cohen SC \& Perucci LCA 2003. Relatório Rede Brasileira de Habitação Saudável - Período: 03/2002 a 07/2003. Documento produzido pela Rede Brasileira de Habitação Saudável. ENSP, Rio de Janeiro.

Cohen SC, Cynamon SC \& Kligerman DC 2003. Versão preliminar da proposta do estudo e desenvolvimento dos padrões regionais de habitabilidade no Brasil 2003 - Rede Brasileira de Habitação Saudável. Documento produzido pela Rede Brasileira de Habitação Saudável. ENSP, Rio de Janeiro.

INHEM 2002. Paquete Docente de Riesgos Físicos. CDRom. INHEM, Havana.

MS. Política Nacional de Promoção da Saúde. Ministério da Saúde, Brasília.

OMS 1998. Promoción de La Salud - Glosario. OMS. Genebra. Disponível em <www.who.org >

OPAS 2000. Guias Metodológicos para Iniciativa de Vivienda Saludable. Disponível em <www.cepis.opsoms.org>

Teixeira MB 1997. Atenção primária ambiental: o foco na experiência local. Debates Sócio-Ambientais 3(8):6-7.

\section{Sites para consulta}

http://www.ensp.fiocruz.br/rbhs

http://www.cepis.ops-oms.org (portal vivienda saludable)

Artigo apresentado em 24/4/2004

Aprovado em 29/6/2004

Versão final apresentada em 15/7/2004 